\title{
Emociones percibidas por los clientes en los encuentros de los servicios para garantizar la lealtad en una relación a largo plazo: Revisión teórica preliminar (Emotions perceived by customers at service encounters to ensure loyalty in a long-term relationship: Preliminary theoretical review)
}

\section{Israel Garza Sepúlveda}

\begin{abstract}
Loyalty remains one of the main issues and concerns for researchers and practitioners. The relationship with satisfaction and service quality is evident, but in turn is still insufficient to achieve. This brings us to keep looking for other relationships and not just a behavioral, attitudinal and cognitive stage but from an emotional level and can achieve loyalty in those dimensions. Service encounters where the employee matches with the customer, is the time where they test all organizational intentions and where the customer receives attention. It is at this point where research has emphasis.
\end{abstract}

Keywords: customers, emotions, service encounters, service loyalty

JEL: $M 120, M 300$

Resumen: La lealtad sigue siendo uno de los principales temas y preocupaciones para los investigadores y practicantes. La relación que tiene con la satisfacción y la calidad del servicio es evidente, pero a su vez sigue estando insuficiente para lograrla. Esto nos lleva a seguir buscando otras relaciones y no solo en una etapa conductual, actitudinal y cognitiva sino desde un plano emocional como podemos lograr la lealtad en esas dimensiones. Los encuentros de servicio, donde coincide el empleado con el cliente, es el momento donde se ponen a prueba todas las intenciones organizacionales y donde el cliente recibe la atención. Es en este momento donde la investigación tiene énfasis.

Palabras clave: clientes, emociones, encuentros de servicio, manejo de personal

\footnotetext{
- Universidad Autónoma de Nuevo León, Facultad de Contaduría Pública y Administración, San Nicolás de los Garza, N.L., México. Email: igzasep@gmail.com
} 


\section{Introducción}

El presente artículo tiene la finalidad de hacer una revisión preliminar de la literatura y hacer una proposición haciendo referencia a la necesidad de seguir desarrollando el tema tanto el contexto como el marco teórico para ampliar en sustento en la relación empírica entre los constructos.

Sector servicios. Vivimos en una economía cada vez más dependiente del sector servicios (Larrea, 1991). El sector representa el $71 \%$ del PIB a nivel mundial según el Banco Mundial, siendo la principal actividad económica del planeta. Esta situación se presenta en países desarrollados como en países emergentes. Los diez países que tienen la mayor concentración del PIB, son Alemania, Brasil, China, Francia, India, Inglaterra, Italia, Japón, Rusia y Estados Unidos, en promedio el sector servicios representa el $68.2 \%$. En particular en México se encuentra en el número 15 , el sector servicios representa el $62 \%$ de su PIB (http://databank.worldbank.org/). Además de su importancia actual también está en crecimiento, en la misma referencia hace un comparativo del año 2000 con el 2014 y en global hubo un incremento del 6\%. El sector industrial, aunque su principal actividad no sea de servicios, también necesita de planeación para el servicio al cliente (Takeuchi \& Quelch, 1983) y es donde se está haciendo un diferenciador.

Los servicios son por mucho el sector más importante y el concepto de administración de servicios apenas comienza e inclusive con confusiones con temas de mercadotecnia y además con muchas áreas de oportunidad (Lovelock, Reynoso, D’Andrea, \& Huete, 2004). El hecho que sea el sector más desarrollado con muchas transacciones lo hace susceptible a caer en malas experiencias para los clientes. La necesidad de seguir investigando en la ciencia de servicio es necesaria para poder abastecer las necesidades crecientes (Osrom, et al., 2010).

Lealtad. Desde el punto de vista de costos de lo que representa la lealtad, siempre va a ser más barato mantener un cliente que traer uno nuevo (Reichheld, 1996). El costo de la publicidad en los medios impresos, digitales, tener personal capacitado para atender a los prospectos, entre muchos otros, es más caro que mantener los clientes actuales y que a través de ellos crecer. El crecimiento puede ser dentro de la misma cuenta o por 
recomendación sin invertir en generar nuevas oportunidades. Inclusive en notas periodísticas como Nielsen (2013) publicó que atributo de calidad en servicio es la principal causa para pensar en cambiar de servicio, solo después del precio.

Emociones. El papel de las emociones es cada vez más utilizado debido a que los modelos cognitivos resultan muy limitados para explicar la satisfacción del cliente y la lealtad (Bigné \& Andreu, 2004).

Las emociones tiene un papel significativo en el comportamiento del cliente (Vázquez Casielles, Suárez Álvarez, \& del Río Lanza, 2009). Las emociones son un factor determinante en cuanto a sus reacciones, donde dependiendo de su estado de ánimo éstas pueden variar (Babin, Darden, \& Babin, 1998). El estado de ánimo positivo también se ha encontrado que favorece la integración de información, promueve las relaciones por estímulos y la búsqueda de soluciones creativas (Isen, Niedenthal, \& Cantor, 1992). La percepción, uso, entendimiento y regulación de las emociones está relacionado con la adaptación de las personas (Mayer \& Salovey, 1997).

El afecto también repercute en la recuperación de la memoria, cuando está en un buen estado de ánimo tiene a tener mejores recuerdos de material positivo que de material negativo o neutro (Isen, Shalker, Clark, \& Karp, 1978) (Teasdale \& Russell, 1983). Las personas en estado de ánimo positivo tienden a evaluar otros estímulos positivamente, en comparación con las que están neutras o negativas (Schwarz \& Clore, 1983). Los estados de ánimo afectan entre otras cosas a la recomendación de boca-en-boca (Westbrook, 1987). Los administradores de empresas de servicios deben asegurarse que sus empleados tengan la competencia emocional suficiente para entender a sus clientes (Delcourt C., Gremler, van Riel, \& van Birgelen, 2013).

\section{Marco teórico}

La lealtad en los productos tiene ciertas características que no puede generalizarse hacia la lealtad en los servicios (Bloemer \& Kasper, 1995), esto nos lleva a buscar información en específico para el sector en estudio. La evolución del constructo de lealtad en los servicios la podemos simplificar en tres grandes etapas. La primera es cuando la variable tenía un enfoque 0 definición acotado al comportamiento del consumidor (Jacoby \& Chestnut, 1978; Tucker, 1964). Como su nombre lo indica solo se observaba eventos 
como la intención de recompra en un tiempo determinado, o que hacer comentarios positivos. En la segunda etapa la caracteriza que además de comportamiento del consumidor se agrega la dimensión actitudinal (Day, 1976) (Javalgi \& Moberg, 1997). La cual consiste en las intenciones de permanecer y confirma sus preferencias. La tercera etapa agrega un apartado más al de comportamiento y actitudinal, la cognitiva como lo describe (Gremler \& Brown, 1996). Esta dimensión se refiere a ser y mantenerse como la primera opción.

La calidad de los servicios es un tema que empieza a tener relevancia en los años ochenta, cuando se empiezan a desarrollar los primeros modelos cualitativos para le medición de las expectativas, percepciones y preponderancia de los servicios. La calidad de los servicios, sigue representando una de los principales temas de las organizaciones.

Las principales características de los servicios son: intangibles, heterogéneos e inseparables (Parasuraman, Zelthaml, \& Berry, 1985). Intangibles debido a que los servicios pueden ser desde una asesoría, la cual no hay un entregable de por medio. Heterogéneo debido a que puede variar entre empleados, clientes y días en los que se dio el servicio. Inseparable se refiere a que la producción y entrega se realiza al mismo tiempo. Estas son una de las principales características de los servicios, algunas no aplicaran para todos pero son las más generales.

La evaluación de los servicios es un proceso completo que no depende solamente de la entrega del mismo sino de todo el proceso que lo interviene (Parasuraman, Zeithaml, \& Berry, 1988). El proceso incluye situaciones, físicas, emocionales, de proceso, participación o no del cliente entre otros factores. Un proceso de compra empieza desde que el cliente tiene el primer contacto con el servicio, desde que se estaciona, por ejemplo, pasa por una experiencia de servicio y no termina hasta que finalmente sale del estacionamiento. La evaluación termina siendo el cumulo de situaciones que la persona experimentó desde su llegada hasta su partida.

Los servicios se caracterizan por las siguientes dimensiones: confiabilidad, empatía, responsabilidad, seguridad y tangibles (Parasuraman, Berry, \& Zeithaml, 1991). Confiabilidad está relacionada con la destreza con la que los empleados entregan el servicio de la forma en que se pactó o se acordó. Empatía está relacionada con la forma en que se cuida al cliente, 
viendo no solo por los intereses de la empresa sino por ambos y por la atención personalizada. Responsabilidad está relacionada por un lado con la buena voluntad que demuestran los empleados a los clientes de ayudarlos en todo lo necesario, por el otro como la prontitud con la que los atienden. Seguridad está relacionada por un lado con el conocimiento y destreza que muestren los empleados, por otro con la cortesía y la habilidad de transmitir confianza al momento de atender a los clientes. Tangibles está relacionado con la parte visual que aprecia el cliente que se utilice para atender y/o brindar el servicio, va desde la apariencia de los empleados, los materiales de comunicación, hasta las instalaciones y equipo en general. Una característica de las dimensiones es que confiabilidad habla sobre la entrega del servicio, mientras las otras sobre del proceso de entrega.

Los clientes tienen cierta flexibilidad con los empleados que los atienden, no esperan que todo sea perfecto, sino que se encuentre dentro de parámetros razonables de servicio. A esta región se le conoce como la zona de tolerancia (Parasuraman, Berry, \& Zeithaml, 1991) y explica que el cliente se encuentra entre el servicio deseado y el adecuado. Sus principales características es que es dinámico y está relacionado con encuentros pasados, lo que lo hace un tema importante a comprender por las organizaciones para siempre estar dentro de los límites. La zona de tolerancia es un espacio en el cual el cliente está cómodo con el servicio y representa un nivel de servicio suficiente para continuar siendo cliente. El reto de las organizaciones es superar las expectativas y no solo llegar al servicio adecuado sino ir más allá sorprendiendo a sus clientes.

Los clientes requieren y solicitan un mayor acercamiento, quieren que la relación sea muy estrecha al grado de mostrar personalización en sus experiencias (Parasuraman, Berry, \& Zeithaml, 1991). El nivel de personalización es una característica del servicio donde se conoce información del cliente como su nombre, fecha de cumpleaños o diferentes conexiones que permitan modificar su experiencia de manera única. El acercamiento se puede hacer de varias formas, como altas de clientes 0 tarjetas de cliente frecuente donde se solicite dicha información, el reto es hacer el mejor uso para beneficio del cliente y su experiencia.

Para favorecer la relación se destaca el entendimiento mutuo entre el empleado y el cliente (Mohr \& Bitner, 1991). El lograr una relación estrecha entre el empleado y el cliente te brinda la oportunidad de tener el dialogo 
suficiente para establecer las prioridades y desarrollar el servicio como se necesita.

Los encuentros de servicio, donde coincide el empleado con el cliente, es el momento donde se ponen a prueba todas las intenciones organizacionales y donde el cliente siente la atención recibida (Davies \& Rosa, 2012). No todos los encuentros de servicio son igual de importantes, debido al tipo de servicio, finalidad y situación es lógico que no tengan el mismo peso o importancia (Bitner, 1995) por lo que se deben identificar y atender de la misma forma.

\section{Método de análisis}

Para el desarrollo de este artículo se buscaron las principales publicaciones y referencias que se tenían disponibles dentro de la UANL, se utilizó la herramienta de Google Scholar para la búsqueda de palabras clave tomando como referencia los trabajos más citados y de autores reconocidos. Una vez que se tuvieron los primeros documentos se buscó ampliar mediante las referencias.

Las delimitaciones del trabajo son variables que tengan relación con un sentido afectivo, por ello se quitaron algunos ítems no relacionados. Las limitaciones son principalmente el acceso a documentos que tiene convenio con la universidad, por lo que no se incluyeron todos los que se encontraron o se tenían como referencia en otros artículos.

\section{Análisis documental y resultados}

A manera de resumen y análisis se desarrolló la operacionalización de las variables, con base en la revisión preliminar de literatura. Para cada una de ellas se establece la definición teórica, su naturaleza y la unidad de medición ver Tabla 1. La variable dependiente es la lealtad de servicio, la cual fue definida por Gremler y Brown (1996, pág. 173), "grado en que un cliente habitual exhibe el comportamiento de compra de un proveedor de servicio, posee una disposición de actitud positiva hacia el proveedor, y considera usar sólo ese proveedor cuando una necesidad de este servicio se plantea". La definición tiene tres dimensiones: lealtad conductual, lealtad actitudinal y 
lealtad cognitiva. Cada una de las dimensiones está relacionada con un total de 12 ítems para su medición en una escala de 7 puntos, donde se establece si está total en desacuerdo hasta totalmente de acuerdo.

\section{Tabla 1. Operacionalización de las variables}

\begin{tabular}{|c|c|c|c|}
\hline Variable & Definición & Naturaleza & Unidad de medición \\
\hline $\begin{array}{c}\text { Lealtad del } \\
\text { servicio } \\
\text { (Variable } \\
\text { dependiente) }\end{array}$ & $\begin{array}{l}\text { "Es el grado en que un cliente } \\
\text { habitual exhibe el } \\
\text { comportamiento de compra } \\
\text { de un proveedor de servicio, } \\
\text { posee una disposición de } \\
\text { actitud positiva hacia el } \\
\text { proveedor, y considera usar } \\
\text { sólo ese proveedor cuando } \\
\text { una necesidad de este } \\
\text { servicio se plantea". }\end{array}$ & $\begin{array}{l}\text { Basado en la } \\
\text { definición de } \\
\text { (Gremler \& } \\
\text { Brown, 1996, } \\
\text { pág. 173) }\end{array}$ & $\begin{array}{c}\text { Instrumento basado en el mismo } \\
\text { autor (Gremler \& Brown, 1996) en un } \\
\text { listado de } 12 \text { aseveraciones y se } \\
\text { contesta en una escala de } 7 \text { puntos } \\
\text { si está totalmente en desacuerdo o } \\
\text { totalmente de acuerdo. } \\
\text { Para tomar solo las relacionadas con } \\
\text { las emociones queda un instrumento } \\
\text { con } 8 \text { ítems. }\end{array}$ \\
\hline $\begin{array}{c}\text { Calidad del } \\
\text { servicio } \\
\text { (variable } \\
\text { independiente) }\end{array}$ & $\begin{array}{l}\text { "La calidad de servicio } \\
\text { percibida es visto como el } \\
\text { grado y la dirección de la } \\
\text { discrepancia entre las } \\
\text { percepciones y expectativas } \\
\text { de los clientes" }\end{array}$ & $\begin{array}{l}\text { Basado en la } \\
\text { definición de } \\
\text { (Parasuraman, } \\
\text { Zeithaml, \& Berry, } \\
\text { 1988, pág. 17) }\end{array}$ & $\begin{array}{c}\text { Instrumento basado en SERVQUAL } \\
\text { con } 21 \text { aseveraciones, } \\
\text { (Parasuraman, Zeithaml, \& Berry, } \\
\text { 1988); información recopilada y } \\
\text { traducida al español por (Castillo } \\
\text { Morales, 2005) } \\
\text { Para tomar solo las relacionadas con } \\
\text { las emociones queda un instrumento } \\
\text { con } 8 \text { ítems. }\end{array}$ \\
\hline $\begin{array}{c}\text { Competencia } \\
\text { emocional del } \\
\text { empleado }\end{array}$ & $\begin{array}{l}\text { "El empleado demostró la } \\
\text { capacidad de percibir, } \\
\text { comprender y regular las } \\
\text { emociones de los clientes en } \\
\text { un encuentro de servicio para } \\
\text { crear y mantener un clima } \\
\text { adecuado para el servicio" }\end{array}$ & $\begin{array}{l}\text { Basado en la } \\
\text { definición de } \\
\text { (Delcourt C. , } \\
\text { Gremler, van } \\
\text { Rierl, \& van } \\
\text { Birgelen, 2015, } \\
\text { pág. 7) }\end{array}$ & $\begin{array}{l}\text { Instrumento basado en el mismo } \\
\text { autor con } 13 \text { aseveraciones en } \\
\text { escala de } 5 \text { puntos (Delcourt C. } \\
\text { Gremler, van Rierl, \& van Birgelen, } \\
\text { 2015) }\end{array}$ \\
\hline $\begin{array}{l}\text { Encuentro de } \\
\text { servicio }\end{array}$ & $\begin{array}{l}\text { "Un encuentro de servicio ha } \\
\text { sido definida como la } \\
\text { interacción directa entre una } \\
\text { operación de servicio y sus } \\
\text { clientes" }\end{array}$ & $\begin{array}{l}\text { Basado en la } \\
\text { definición de } \\
\text { (Davies \& Rosa, } \\
\text { 2012, págs. 663- } \\
\text { 683) }\end{array}$ & $\begin{array}{l}\text { Instrumento con } 31 \text { aseveraciones en } \\
\text { escala de } 5 \text { puntos (Prince, Arnould, } \\
\text { \& Tuerney, 1995). } \\
\text { Para tomar solo las relacionadas con } \\
\text { las emociones queda un instrumento } \\
\text { con } 8 \text { items. }\end{array}$ \\
\hline
\end{tabular}

La variable independiente de Calidad del servicio, la cual fue definida por (Parasuraman, Zeithaml, \& Berry, 1988, pág. 17), "La calidad de servicio percibida es visto como el grado y la dirección de la discrepancia entre las 
percepciones y expectativas de los clientes". La definición tiene 5 dimensiones, tangibles, confiabilidad, responsabilidad, empatía y seguridad. Cada una de las dimensiones está relacionada con un total de 21 ítems para su medición en una escala de 7 puntos, donde se establece si está total en desacuerdo hasta totalmente de acuerdo. De éstas se tomaron dos dimensiones que están relacionadas con las emociones del servicio. La empatía, la cual está definida como "la capacidad de brindar cuidado y atención personalizada a sus clientes" y la seguridad que está definida "como el conocimiento de los empleados sobre lo que hacen, su cortesía y su capacidad de transmitir confianza". Cada una de las dimensiones está relacionada con un total de 8 ítems para su medición.

La variable independiente de Competencia Emocional del Empleado, la cual fue definida por (Delcourt C., Gremler, van Rierl, \& van Birgelen, 2015, pág. 7), "El empleado demostró la capacidad de percibir, comprender y regular las emociones de los clientes en un encuentro de servicio para crear y mantener un clima adecuado para el servicio". La definición tiene tres dimensiones, percepción de las emociones de los clientes, entendimiento de las emociones de los clientes y regulación de las emociones de los clientes. Cada una de las dimensiones está relacionada con un total de 13 ítems para su medición en una escala de 5 puntos, donde se establece si está total en desacuerdo hasta totalmente de acuerdo.

La variable independiente de encuentros de servicio, la cual fue definida por (Davies \& Rosa, 2012, págs. 663-683) "Un encuentro de servicio ha sido definida como la interacción directa entre una operación de servicio y sus clientes". La definición tiene tres dimensiones, duración, contenido afectivo y proxémica. Cada una de las dimensiones está relacionada con un total de 31 ítems para su medición. De éstas se tomó la dimensión de contenido afectivo por ser la relacionada con emociones. Esta dimensión está relacionada con un total de 8 ítems para su medición.

\section{Conclusiones}

Las emociones tienen un rol fundamental en las entregas de servicio y la proposición es que tenga relación con la lealtad de servicio. En base a la 
literatura pudimos observar que el estado de ánimo afecta la forma en la que pensamos, actuamos y evaluamos a nuestro entorno.

Se invita a continuar investigando en el tema para ampliar el contexto y marco teórico para sumar relaciones empíricas que permitan el soporte para realizar un estudio cuantitativo.

\section{Referencias}

Babin, B., Darden, W., \& Babin, L. (1998). Negative emotions in marketing research: affect or artifact? Journal of Business Research, 42(3), 271-285.

Bigné, E. \& Andreu, L. (2004). Emociones, satisfacción y lealtad del consumidor en entornos comerciales. Distribución y Consumo, 76, 77-87.

Bitner, M. (1995). Building service relationships: It's all about promises. Journal of the Academy of Marketing Science, 23(4), 246-251.

Bloemer, J. M. \& Kasper, H. D. (1995). The complex relationship between consumer satisfaction and brand loyalty. Journal of Economic Psychology, 16(2), 311-329.

Castillo, E. (2005). Escala Multidimensional SERVQUAL. Facultad de Ciencias Empresariales Universidad del Bío-Bío, Chile.

CLELAC. (2013). La industria de electrodomésticos en México. Recuperado el 20 de mayo de 2014 de: http://www.clelac.org.mx/nacional.php

Davies, G. \& Rosa, C. (2012). Employee as symbol: stereotypical age effects on corporate brand associations. European Journal of Marketing, 46(5), 663-683.

Day, G. S. (1976). A two-Dimensional concept of brand loyalty. Journal of Advertising Research, 9, 29-36.

Delcourt, C., Gremler, D., van Riel, A. \& van Birgelen, M. (2013). Effects of perceived employee emotional competence on customer satisfaction and loyalty. The mediating role of rapport. Journal of Service Management, 24(1), 5-24.

Delcourt, C., Gremler, D., van Rierl, A. \& van Birgelen, M. (2015). Employee emotional competence: Construct conceptualization and validation of a customer-based measure. Journal of Service Research, 19(1), 72-87.

Gremler, D. \& Brown, S. W. (1996). Gremler, D. D., \& Brown, S. W. (1996). Service loyalty: its nature, importance, and implications. In Edvardsson, B., Brown, S. W., Johnston, R. \& Scheuing, E. B. (eds), QUIS 5 Advancing service quality: A global perspective, New Jersey: International Service Quality Association, 171-181.

Isen, A. M., Niedenthal, P. M. \& Cantor, N. (1992). An influence of positive affect on social categorization. Motivation and Emotion, 16(1), 65-78.

Isen, A. M., Shalker, T. E., Clark, M. \& Karp, L. (1978). Affect, accessibility of material in memory, and behavior: A cognitive loop? Journal of Personality and Social Psychology, 36(1), 1-12.

Jacoby, J., \& Chestnut, R. W. (1978). Brand loyalty: Measurement and management. New York: John Wiley and Sons, Inc. 
Javalgi, R. G. \& Moberg, C. R. (1997). Service loyalty: Implications for service providers. The Journal of Services Marketing, 11(3), 165-179.

Larrea, P. (1991). Calidad del servicio del marketing a la estrategia. Madrid: Ediciones Díaz de Santos, S. A.

Lovelock, C., Reynoso, J., D’Andrea, G. \& Huete, L. (2004). Administración de servicios; estrategias de marketing, operaciones y recursos humanos. Naucalpan de Juárez: Pearson Education, Inc.

Mayer, J. \& Salovey, P. (1997). What is emotional intelligence? En P. Salovey, \& D. Slayter, Emotional Development and Emotional Intelligent, New York: Basic Books, 3-31.

Mohr, L. \& Bitner, M. (1991). Mutual Understanding Between Customers and Employees in Service Encounters. Advances in Consumer Research, 18(1), 611-617.

Nielsen (2013). How loyal are your customers? Nielsen Reports. Obtenido de http://www.nielsen.com/us/en/insights/reports/2013/how-loyal-are-your-customers.html

Osrom, A. L., Bitner, M. J., Brown, S. W., Burkhard, K. A., Goul, M., Smith-Daniels, V. \& Rabinovich, E. (2010). Moving forward and making a difference: Research priorities for the science of service. Journal of Service Research, 13(1), 4-36.

Parasuraman, A., Berry, L. \& Zeithaml, V. A. (1991). Understanding customer expectations of service. Sloan Management Review, 32(3), 39-48.

Parasuraman, A., Zeithaml, V. \& Berry, L. (1988). SERVQUAL: A Multiple-item scale for measuring consumer perceptions of service quality. Journal of Retailing, 64(1), 12-40.

Parasuraman, A., Zelthaml, V. \& Berry, L. (1985). A conceptual model of service quality and its implications for future research, The Journal of Marketing, 49(4), 41-50.

Price, L., Arnould, E. \& Tuerney, P. (1995). Going to extremes: Managing service encounters and assessing provider performance. The Journal of Marketing, 59(2), 83-97.

Reichheld, F. (1996). The loyalty effect: The hidden force behind growth, profits, and lasting value. Boston: Harvard Business School Press.

Schwarz, N., \& Clore, G. (1983). Mood, misattribution, and judgments of well-being: Informative and directive functions of affective states. Journal of Personality and Social Psychology, 45(3), 513-523.

Takeuchi, H. \& Quelch, J. A. (1983). Quality is more than making a good product. Harvard Business Review, 61(4), 139-145.

Teasdale, J. \& Russell, M. (1983). Differential effects of induced mood on the recall of positive, negative and neutral words. British Journal of Clinical Psychology, 22(3), 163-171.

Tucker, W. T. (1964). The development of brand loyalty. Journal of Marketing Research, 1 , $32-35$.

Vázquez Casielles, R., Suárez Álvarez, L., \& del Río Lanza, A. (2009). Evaluación cognitiva y afectiva de las estrategias de recuperación del servicio: relaciones entre justicia percibida, emociones y satisfacción. Revista Europea de Dirección y Economía de la Empresa, 18(1), 31-50.

Westbrook, R. A. (1987). Product/consumption-based affective responses and postpurchase processes. Journal of Marketing Research, 24(3), 258-270.

\section{Garza}

